\title{
Supermassive black holes in spiral galaxies
}

\author{
Duccio Macchetto ${ }^{1}$ \\ ${ }^{1}$ ESA and Space Telescope Science Institute, 3700 San Martin Drive, Baltimore, MD 21218, \\ USA email: macchetto@stsci.edu
}

\begin{abstract}
We have embarked in an $H S T$ program to determine the masses of black holes in spiral galaxies directly by measuring the line emission arising from an extended accretion disk. For each of the galaxies in our sample we have measured the rotation curve and determined the mass distribution within the inner 5-50 pc. We have modeled the stellar mass component using the photometric data from existing $H S T$ images and using both data sets we have derived the masses of the black holes in each galaxy. These results will be very important in clarifying the role of the black hole in powering the AGN, will shed light into the effectiveness of the accretion mechanisms and finally will be important in addressing the fundamental issue of unification for Seyfert 1 and Seyfert 2 galaxies.
\end{abstract}

\section{Introduction}

It is widely accepted that Active Galactic Nuclei (AGN) are powered by accretion of matter onto massive black holes. AGN activity peaked at $z \sim 1-2$ (e.g., Maloney \& Petrosian 1999) and the high $\left(>10^{12} \mathrm{~L}_{\odot}\right)$ luminosities of quasi stellar objects (QSOs) are explained by accretion onto super massive $\left(\sim 10^{8}-10^{10} \mathrm{M}_{\odot}\right)$ black holes at or close to the Eddington limit. The observed evolution of the space density of AGN (Chokshi \& Turner 1992, Faber et al. 1997, Marconi \& Salvati 2001) implies that a significant fraction of luminous galaxies must host black holes, relics of past activity. Indeed, it is now clear that a large fraction of hot spheroids contain a massive BH (e.g., Magorrian et al. 1998; van der Marel 1999), and it appears that the BH mass is proportional to both the mass of the host spheroid (Kormendy \& Richstone 1995) and its velocity dispersion (Ferrarese \& Merritt 2001, Gebhardt et al. 2000, Merritt \& Ferrarese 2001).

Several radio-galaxies, all associated with giant elliptical galaxies, like M87 (Macchetto et al. 1997), M84 (Bower et al. 1998), NGC 7052 (van der Marel \& van den Bosch 1998) and Centaurus A (Marconi et al. 2001), are now known to host supermassive $\left(\sim 10^{8}-10^{9} \mathrm{M}_{\odot}\right)$ BHs in their nuclei. The luminosity of their optical nuclei indicates that they are accreting at a low rate and/or low accretion efficiency (Chiaberge, Capetti \& Celotti 1999). They presumably sustained quasar activity in the past but at the present epoch are emitting much below their Eddington limits $\left(\mathrm{L} / \mathrm{L}_{\mathrm{Edd}} \sim 10^{-5}-10^{-7}\right)$. The study of the Seyfert $\mathrm{BH}$ mass distribution provides a statistical method of investigating the interplay between accretion rate and $\mathrm{BH}$ growth. In order to achieve this it is necessary to directly measure the BH masses in Seyfert galaxies and to compare their Eddington and Bolometric luminosities using the hard X-ray luminosities. Similarly important will be the comparison between the BH masses found in Seyfert galaxies with those of non active galaxies. However, to date, there are very few secure BH measurements or upper limits in spiral galaxies. It is therefore important to directly establish how common are $\mathrm{BHs}$ in spiral galaxies and whether they follow the same $M_{\mathrm{BH}}-M_{\mathrm{sph}}, M_{\mathrm{BH}}-\sigma_{\star}$ correlations as Elliptical galaxies.

To detect and measure the masses of massive BHs requires spectral information at the highest possible angular resolution - the "sphere of influence" of massive BHs is 
typically $\sim 1^{\prime \prime}$ in radius even in the closest galaxies. Nuclear absorption line spectra can be used to demonstrate the presence of a $\mathrm{BH}$, but the interpretation of the data is complex because it involves stellar-dynamical models that have many degrees of freedom. In Seyfert galaxies the problems are compounded by the copious light from the AGN. Studies at $H S T$ resolution of ordinary optical emission lines from gas disks in principle provide a more widely applicable and readily interpreted way of detecting BHs (cf. M87, Macchetto et al. 1997, Barth et al. 2001) provided that the gas velocity field are not dominated by non gravitational motions.

\section{The Sample, Observational Strategy and Modeling}

Prompted by these considerations, we have undertaken a spectroscopic survey of 54 spirals using STIS on the Hubble Space Telescope. Our sample was extracted from a comprehensive ground-based study by Axon et al. who obtained $\mathrm{H} \alpha$ and N II rotation curves at a seeing-limited resolution of 1", of $128 \mathrm{Sb}, \mathrm{SBb}, \mathrm{Sc}$, and $\mathrm{SBc}$ spiral galaxies from RC3. By restricting ourselves to galaxies with recession velocities $V<2000 \mathrm{~km} / \mathrm{s}$, we obtained a volume-limited sample of 54 spirals that are known to have nuclear gas disks and span wide ranges in bulge mass and concentration. The systemic velocity cutoff was chosen so that we can probe close to the nuclei of these galaxies, and detect even lower-mass black holes. The frequency of AGN in our sample is typical of that found in other surveys of nearby spirals, with comparable numbers of weak nuclear radio sources and LINERS.

The observational strategy, used for all the galaxies in our sample, consisted in obtaining spectra at three parallel positions with the central slit centered on the nucleus and the flanking ones at a distance of 0.2 . At each slit position we obtained two spectra with the G750M grating centered at $\mathrm{H} \alpha$, with the second spectrum shifted along the slit by an integer number of detector pixels in order to remove cosmic-ray hits and hot pixels. The nuclear spectrum (NUC) was obtained with the $0{ }^{\prime \prime} 1$ slit and no binning of the detector pixels, yielding a spatial scale of $0 . \prime 0507 /$ pix along the slit, a dispersion per pixel of $\Delta \lambda=0.554 \AA$ and a spectral resolution of $\mathcal{R}=\lambda /(2 \Delta \lambda) \simeq 6000$. The off-nuclear spectra (POS1 and POS2) were obtained with the $0{ }^{\prime \prime} 2$ slit and $2 \times 2$ on-chip binning of the detector pixels, yielding $0^{\prime \prime} 101 /$ pix along the slit, $1.108 \AA /$ pix along the dispersion direction and $\mathcal{R} \simeq 3000$. The raw spectra were processed with the standard pipeline reduction software.

We derived rotation curves for each of the observed slit positions and applied our modeling code, described in detail in Marconi et al. (2003), to fit the observed rotation curves. Briefly the code computes the rotation curves of the gas assuming that the gas is rotating in circular orbits within a thin disk in the galaxy potential. The gravitational potential has two components: the stellar potential, determined from WFPC or NICMOS observations and characterized by its mass-to-light ratio, and a dark mass concentration (the black hole), spatially unresolved at $H S T+$ STIS resolution and characterized by its total mass $\mathrm{M}_{\mathrm{BH}}$. In computing the rotation curves we take into account the finite spatial resolution of $H S T+$ STIS and we integrate over the slit and pixel area. The $\chi^{2}$ is minimized to determine the free parameters using a downhill simplex algorithm . The emission line surface brightness is modeled with a composition of two gaussians, the first reproducing the central emission peak while the second accounts for the brightness behaviour at large radii. Having fixed the line brightness distribution, the free parameters of the fit are the systemic velocity, $v_{\text {sys }}$, the impact parameter (i.e., the distance between the slit center and the center of rotation) $b$, the position of the galaxy center along the slit $s_{0}$, the angle between the slit and the line of nodes, $\theta$, the disk inclination $i$, the mass 


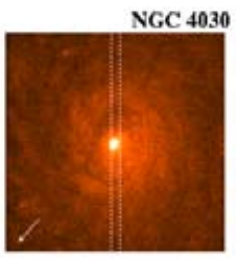

NGC 4051

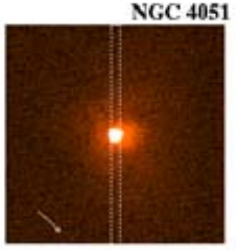

NGC 4100

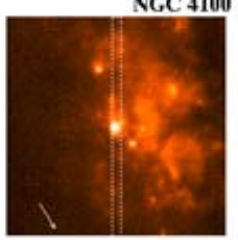

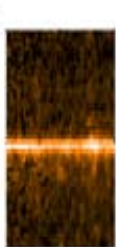
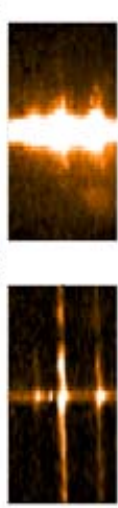

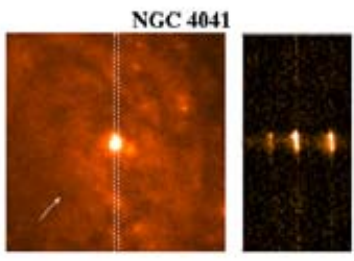

NGC 4088

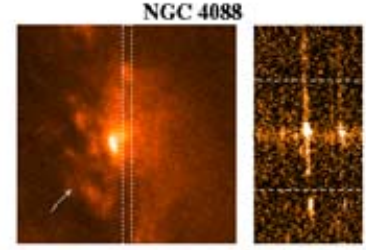

NGC 4212
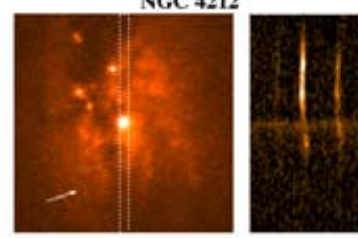

Figure 1.

to light ratio, $\Upsilon$ and the black hole mass $M_{\mathrm{BH}}$. We perform a $\chi^{2}$ minimization allowing all parameters to vary freely.

In this review I discuss the key results for two galaxies in our sample, namely NGC 4041 and NGC 5252, while in Fig. 1 I show a sample of the images and spectra for a subset of the other galaxies that we have observed.

\section{NGC 4041}

NGC 4041 is classified as a Sbc spiral galaxy with no detected AGN activity. Its average heliocentric radial velocity from radio measurements is $1227 \pm 9 \mathrm{~km} \mathrm{~s}^{-1}$ becoming $\simeq 1480 \mathrm{~km} \mathrm{~s}^{-1}$ after correction for Local Group infall onto Virgo. With $\mathrm{H}_{0}=75 \mathrm{~km} \mathrm{~s}^{-1}$ $\mathrm{Mpc}^{-1}$ this corresponds to a distance of $\simeq 19.5 \mathrm{Mpc}$ and to a scale of $95 \mathrm{pc} / \mathrm{arcsec}$.

The HST/STIS spectra were used to map the velocity field of the gas in its nuclear region. We detected the presence of a compact $(r \simeq 0$.' $4 \simeq 40 \mathrm{pc}$ ), high surface brightness, circularly rotating nuclear disk cospatial with a nuclear star cluster. This disk is characterized by a rotation curve with a peak to peak amplitude of $\sim 40 \mathrm{~km} \mathrm{~s}^{-1}$ and is systematically blueshifted by $\sim 10-20 \mathrm{~km} \mathrm{~s}^{-1}$ with respect to the galaxy systemic velocity.

The standard approach followed in gas kinematical analysis is to assume that (i) gas disks around black holes are not warped i.e., they have the same line of nodes and inclinations as the more extended components, and (ii) the stellar population has a constant mass-to-light ratio with radius (e.g., van der Marel \& van den Bosch ${ }^{-1} 998$; Barth et al. 2001). Using the emission line flux distribution derived from the imaging data, the inclination of the galactic disk can be fixed to $i=20^{\circ}$, i.e., The remaining free parameters can then be the fit with the procedure described earlier and we find that, in order to reproduce the observed rotation curve, a dark point mass [supermassive BH] of $\left(1_{-0.7}^{+0.6}\right) \times 10^{7} \mathrm{M}_{\odot}$ is needed. However, the blueshift of the inner disk suggests the 


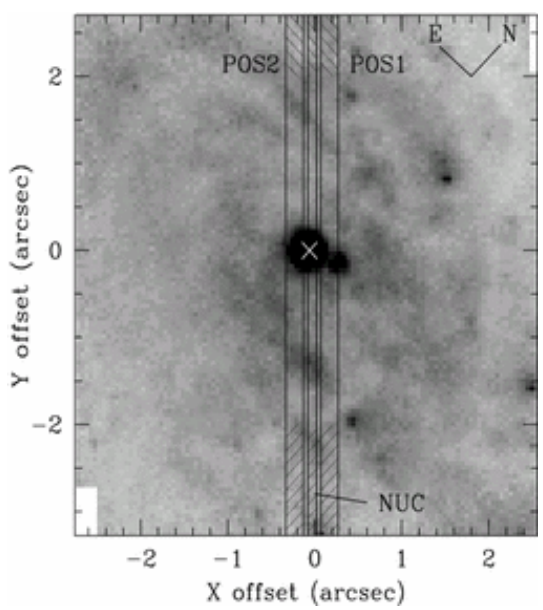

Figure 2. Slit positions overlaid on the acquisition image. The 0,0 position is the position of the target derived from the STIS ACQ procedure. The white cross is the kinematic center derived from the fitting of the rotation curves.
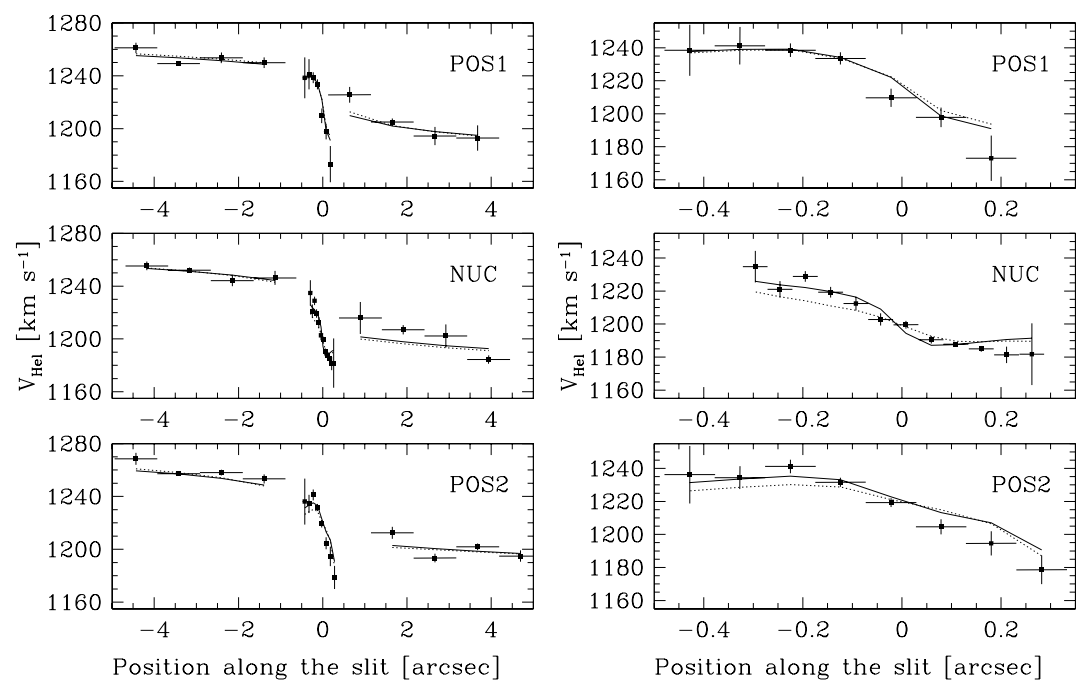

Figure 3. Best fit standard model of the observed rotation curves (solid line) compared with the data. The dotted line is the best fit model without a black hole. The model values are connected by straight lines in order to guide the eye. Note that points from external and nuclear regions are not connected because they are kinematically decoupled. The right panel is a zoom on the nuclear disk region.

possibility that the nuclear disk could be dynamically independent. Following this line of reasoning we have relaxed the standard assumptions and model the curves by allowing variations in the stellar mass-to-light ratio and the disk inclination. We have found that the kinematical data can be accounted for by the stellar mass provided that either the mass-to-light ratio is increased by a factor of $\sim 2$ or the inclination is allowed to vary. This model resulted in a $3 \sigma$ upper limit of $6 \times 10^{6} \mathrm{M}_{\odot}$ for the mass of any nuclear black hole.

Combining the results from the standard and alternative models, the present data only allow us to set an upper limit of $2 \times 10^{7} \mathrm{M}_{\odot}$ to the mass of the nuclear $\mathrm{BH}$. 


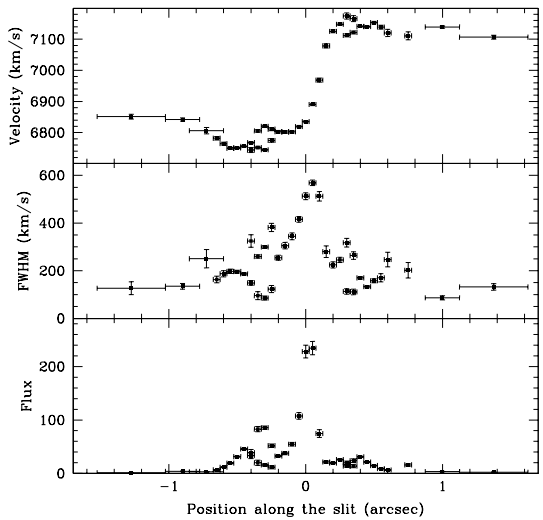

Figure 4. NGC5252. Velocity, flux and FWHM at the NUC (center) slit.

If this upper limit is taken in conjunction with an estimated bulge B magnitude of -17.7 and with a central stellar velocity dispersion of $\simeq 95 \mathrm{~km} \mathrm{~s}^{-1}$, the putative black hole in NGC 4041 is not inconsistent with both the $M_{\mathrm{BH}}-L_{\mathrm{sph}}$ and the $M_{\mathrm{BH}}-\sigma_{\star}$ correlations.

\section{NGC 5252}

NGC 5252 is an early type (S0) Seyfert 2 galaxy at a redshift $z=0.023$ whose line emission shows a biconical morphology (Tadhunter \& Tsvetanov 1989) extending out to $20 \mathrm{kpc}$ from the nucleus along $\mathrm{PA}-15^{\circ}$. On a sub-arcsec scale three emission line knots form a linear structure oriented at PA $\sim 35$, close to the bulge major axis, suggestive of a small scale gas disk. For $\mathrm{H}_{\mathrm{o}}=75 \mathrm{Km} \mathrm{s}^{-1} \mathrm{Mpc}^{-1}$ at the distance of NGC 5252 (92 Mpc), $1^{\prime \prime}$ corresponds to $450 \mathrm{pc}$.

Fig. 4 shows the line central velocity, flux and FWHM for the central slit of our STIS observations. Emission, which is detected out to a radius of $\sim 1$ 1". 6 corresponding to $\sim 720 \mathrm{pc}$, is strongly concentrated showing a bright compact knot cospatial with the continuum peak. Two secondary emission line maxima are also present at $\pm 0.35^{\prime \prime}$ from the main peak. They represent the intersection of the slit with emission line knots seen in our WFPC and STIS images. Two different gas systems are present in the nuclear regions of NGC 5252: the first shows a symmetric velocity field, with decreasing line width and can be interpreted as being produced by gas rotating around the nucleus. The second component, showing significant non circular motions, is found to be associated exclusively with the off-nuclear blobs.

Following the fitting procedure described earlier, the best fit to our data is obtained for a black hole mass $M_{\mathrm{BH}}=9.5 \times 10^{8} \mathrm{M}_{\odot}$. The model fitting of the nuclear rotation curve of NGC 5252 shows that the kinematics of gas in its innermost regions can be successfully accounted for by circular motions in a thin disk when a point-like dark mass (presumably a supermassive black hole) is added to the galaxy potential.

The central velocity dispersion of NGC 5252 (Nelson \& Whittle, 1995) is $190 \pm$ $27 \mathrm{~km} \mathrm{~s}^{-1}$. The correlation between velocity dispersion and black hole mass predicts a mass of $M=1.0_{-0.5}^{+1.0} 10^{8} \mathrm{M}_{\odot}$ where the error is dominated by the uncertainty in $\sigma_{c}$. Therefore, the black hole mass we derived for NGC 5252 is larger by a factor $\sim 10$ than the value expected from this correlation! (see Fig. 5). This value however is in good agreement with the correlation between bulge and $\mathrm{BH}$ mass. As for its active nucleus, NGC 5252 is an outlier when compared to the available data for Seyfert galaxies, not 

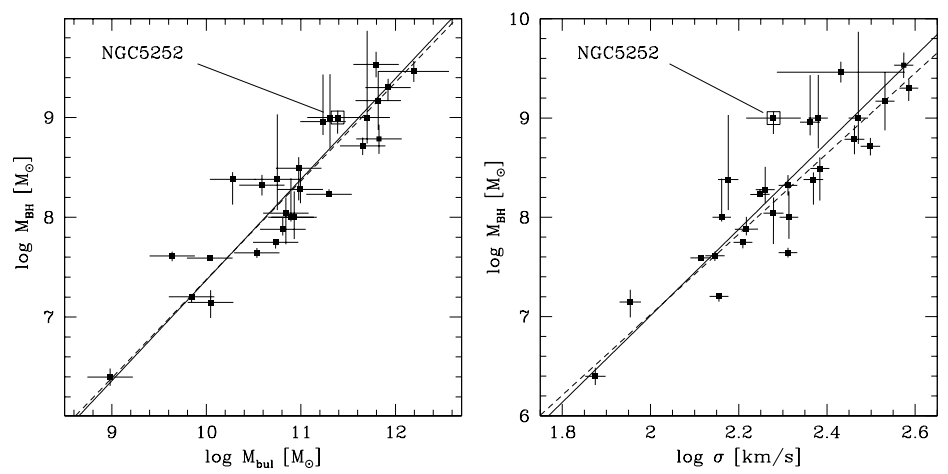

Figure 5. Left, $\mathrm{M}_{\mathrm{BH}}$ vs. bulge mass and right, $\mathrm{M}_{\mathrm{BH}}$ vs. stellar velocity dispersion $\sigma_{e}$ with the best fits obtained from a bisector linear regression analysis (solid line) and ordinary least-square (dashed line).

only as it harbours a black hole larger than typical for these objects, but also as its host galaxy is substantially brighter than average for Seyfert galaxies. On the other hand, both the black hole and the bulge's mass are typical of the range for radio-quiet quasars. Combining the determined $\mathrm{BH}$ mass with the hard X-ray luminosity, we estimate that NGC 5252 is emitting at a fraction $\sim 0.005$ of $\mathrm{L}_{\mathrm{Edd}}$. This active nucleus thus appears to be a quasar relic, now probably accreting at a relatively low rate, rather than a low black hole mass counterpart of QSOs.

\section{Acknowledgements}

The work described here is the result of a long standing collaboration with David Axon, Alessandro Capetti and Alessandro Marconi. They deserve the credit for the results.

\section{References}

Barth, A. J., Sarzi, M., Rix, H., Ho, L. C., Filippenko, A. V., \& Sargent, W. L. W. 2001, ApJ, 555,685

Bower, G. A., et al. 1998, ApJ, 492, L111

Chiaberge, M., Capetti, A., \& Celotti, A. 1999, A\&A, 349, 77

Chokshi, A., \& Turner, E. L. 1992, MNRAS, 259, 421

Faber, S. M., et al. 1997, AJ, 114, 1771

Ferrarese, L., \& Merritt, D. 2000, ApJ, 539, L9

Gebhardt, K., et al. 2000, ApJ, 539, L13

Kormendy, J., \& Richstone, D. 1995, ARA\&A, 33, 581

Macchetto, F., Marconi, A., Axon, D. J., Capetti, A., Sparks, W., \& Crane, P. 1997, ApJ, 489, 579

Magorrian, J., et al. 1998, AJ, 115, 2285

Maloney, A., \& Petrosian, V. 1999, ApJ, 518, 32

Marconi, A., Capetti, A., Axon, D. J., Koekemoer, A., Macchetto, D., \& Schreier, E. J. 2001, ApJ, 549, 915

Marconi, A., \& Salvati, M. 2001, astro-ph/0110698

Marconi, A., et al. 2003, ApJ, 586, 868

Merritt, D., \& Ferrarese, L. 2001, ApJ, 547, 140

Nelson, C. H., \& Whittle, M. 1995, ApJS, 99, 67

Tsvetanov, Z. I., Morse, J. A., Wilson, A. S., \& Cecil, G. 1996, ApJ, 458, 172

van der Marel, R. P. 1999, In Galaxy Interactions at Low and High Redshift IAU Symp. 186, p. 333

van der Marel, R. P., \& van den Bosch, F. C. 1998, AJ, 116, 2220 